\title{
Preface: special topic on fluorescent chemical/biological sensors and imaging
}

\author{
Shu Wang ${ }^{1} \&$ Yu Fang ${ }^{2}$ \\ ${ }^{1}$ Key Laboratory of Organic Solids; Institute of Chemistry, Chinese Academy of Sciences, Beijing 100190, China \\ ${ }^{2}$ Key Laboratory of Applied Surface and Colloid Chemistry (Ministry of Education); School of Chemistry and Chemical Engineering, \\ Shaanxi Normal University, Xi'an 710062, China
}

Citation: Wang S, Fang Y. Preface: special topic on fluorescent chemical/biological sensors and imaging. Sci China Chem, 2016, 59: 1-2, doi: $10.1007 / \mathrm{s} 11426-015-5546-\mathrm{z}$

There is currently considerable interest in the development of fluorescent molecular probes for use in sensitive sensing and imaging applications. The design, synthesis and application of fluorescent molecular probes that selectively and specifically respond to the presence of a specific analyte is a multidisciplinary research area involving numerous fields of modern science and medicine, including molecular biology, chemical biology, biophysics, clinical diagnosis and analytical/environmental chemistry, etc. For sensing application, advances in the understanding of molecular probe structure have enabled the design of systems that detect a variety of chemical, biological, or physical stimuli, such as metal ions, small active molecules, DNA, RNA and protein. Fluorescent probes include not only small molecular dyes but also polymers and nanoparticles that can present multiple reporting groups and thus produce large changes in fluorescent signals. Another rapidly evolving application of fluorescent probes is molecular imaging. Molecular imaging has the ability to visualize and quantitatively measure the function of biological and cellular processes in vivo, and it actually plays a major role in understanding physiological processes. Furthermore, fluorescent probes can also be used for medical imaging, which is critical for diagnosis, surgical, guidance/follow-up, and treatment monitoring, etc. Compared with other techniques, fluorescent imaging is more attractive because it possesses a number of superiorities, such as real-time imaging, relatively inexpensive, higher spatial resolution, and involving no radiation source. In addition, the imaging can be conducted in vivo, which does not require invasive biopsies or surgical procedures. Key challenges in fluorescent sensing and imaging are to improve the sensitivity (brightness), selectivity and long-term stability of the sensing and imaging systems, particularly in complex and hostile environments, as well as applications for disease management, and pathogen identification.

The objective of this special topic of Science China Chemistry is to provide the readership with a concise collection of 12 articles focused on chemical and biological sensors and imaging. Each paper is authored by a leading group in this research area. The collection includes 8 focused research articles that highlight the development of new chemical, biological and nanoparticle probes that are useful for chemical and biological sensing, one-photon and two-photon cell imaging and photodynamic applications. In addition, the special topic also contains 4 Review articles, which highlight the very recent progress in the field of thin film fluorescent probes, SERS-based detections, singlebacteria analysis by flow cytometry and photosensitizers for photodynamic therapy, respectively. We hope that the readers together with us enjoy reading these articles. We thank all authors and reviewers for their dedicated and efficient work. We also thank the editors, Dr. Junjian Xu and Ms. Li $\mathrm{Fu}$, for their hard work to make this special topic possible.

Corresponding authors (email: wangshu@iccas.ac.cn; yfang@snnu.edu.cn) 


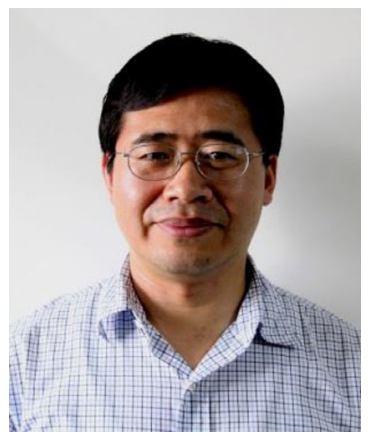

Prof. Shu Wang received his B.S. degree from Department of Chemistry, Hebei University in 1994, and Ph.D. degree in organic synthesis at Department of Chemistry, Peking University in 1999. Following two years of postdoctoral research at Institute of Chemistry, Chinese Academy of Sciences, he moved to Institute of Polymers and Organic Solids, University of California at Santa Barbara to continue his postdoctoral research. In 2004, he became a professor of Institute of Chemistry, Chinese Academy of Sciences. His current research interests include design, synthesis and properties of organic conjugated molecules, biosensors, cell imaging, disease therapeutics and chemical biology.

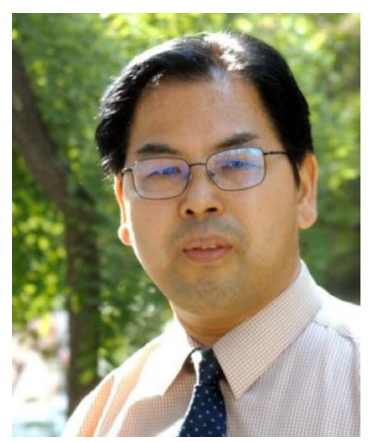

Prof. Yu Fang received his B.S. degree from Shaanxi Normal University (Xi'an, China), M.S. degree from Central China Normal University (Wuhan, China), and Ph.D. degree from Lancaster University (Lancaster, UK) under the mentorship of professor Ian Soutar. He spent one and a half years as a visiting scholar with professor John F. Kennedy at Birmingham University (Birmingham, UK). He is a senior professor of physical chemistry and polymer science of Shaanxi Normal University. His research interests include investigations of functional fluorescent films, stimulusresponsive molecular gels, and exploration of their potential applications. 